\title{
Tuberculosis Diagnosis
}

\author{
Abdollah Karimi ${ }^{1}$; Roxana Mansour Ghanaie ${ }^{1{ }^{1 *} ;}$; Farideh Shiva ${ }^{1}$ \\ ${ }^{1}$ Pediatric Infections Research Center, Mofid Children's Hospital, Shahid Beheshti University of Medical Sciences, Tehran, IR Iran \\ *Corresponding author: Roxana Mansour Ghanaie, Pediatric Infections Research Center, Mofid Children's Hospital, Shahid Beheshti University of Medical Sciences, Tehran, IR Iran. \\ Tel:+98-2122226941, Fax: +98-2122226941, E-mail: roxanaghanaie@yahoo.com \\ Received: May 23, 2014; Accepted: June 8, 2014
}

Keywords:Tuberculosis; WHO

Tuberculosis (TB) is one of the main single infectious agents, which causes death worldwide. It is estimated that $95 \%$ of more than eight million new cases of TB and $99 \%$ of two million deaths each year occur in middleand low-income countries (1). The risk of death in patients with Human Immunodeficiency Virus (HIV) and TB is twice more than those with HIV. Tuberculosis is responsible for $25 \%$ of all avoidable deaths in countries with limited resources (2).

According to a report by the World Health Organization (WHO), the prevalence and incidence of TB in Iran were 33 (13-61) and 21 (15-28) per 100000 population in 2012 (3), among which $43 \%$ of the new cases were smear-positive, $20 \%$ smear negative, $5 \%$ smear-unknown and $31 \%$ extra pulmonary; $7 \%$ of the new TB cases and $27 \%$ of retreatment cases were tested for multidrug-resistant TB (MDR) among which 5\% (3.4-7) and 48\% (35-62) were found positive, respectively (3). Tuberculosis in children differs from those of adults from various aspects such as immunological and pathophysiological responses that affect the prevention, diagnosis and treatment methods in children (4).

In addition, since there is no cure for some strains of MDR Tuberculosis, it is crucial to prevent the MDR strains emergence, properly control and eliminate transmission of TB infection which highlight early diagnosis, use of rapid diagnostic methods with high sensitivity, specificity, and prompt treatment (5). Diagnosis of TB is based on thorough assessment of all the findings derived from epidemiology, background (including TB contacts history and TB symptoms), clinical examination and relevant investigations like chest radiography, tuberculin skin test, and sputum smear microscopy.

The commonest symptoms are chronic unremitting cough, fever, and weight loss (4). Tuberculosis can be considered as a possible diagnosis in anyone with intermittent fever and other unexplained subacute symptoms especially when there is no response to ordinary antibiotic therapy (6). Cases with compatible signs and symptoms are considered as suspected Tuberculosis. In order to con- firm Tuberculosis, it is necessary to detect tubercle bacilli by routine microbiologic tests.

Bacteriological confirmation of TB, for example sputum microscopy in children with pulmonary TB who are old enough to produce a sputum sample and histology from different specimens should be sought whenever possible. Case detection through quality-assured bacteriology is one of the components of The stop TB strategy (4). Accordingly, multiple samples of sputum or gastric lavage should be examined; it is more accurate than bronchoalveolar lavage and has positive yield in $30 \%$ to $40 \%$ of the patients. All of the obtained specimens should be sent for mycobacterial culture whenever possible. Traditional staining and culture include Ziehl-Neelsen and Egg-based/agar-based media such as Lowenstein-Jensen medium, and Middlebrook agar was performed. Modified methods, if feasible, increase sensitivity. Culture is not only more sensitive than smear, but it is also the only way to differentiate Mycobacterium tuberculosis from other non-tuberculous mycobacteria. A bacteriological diagnosis is especially important for children with at least one of the following symptoms: suspected drug resistance, HIV infection, complicated or severe cases of disease, an uncertain diagnosis (4). A trial of treatment with TB medications is not generally recommended to diagnose $\mathrm{TB}$ in children, unless there is a life-threatening condition. In situations with no access to new laboratory tests, anti-tuberculous treatment is started in pediatric practice if three of the following five criteria are present:

1) Compatible signs and symptoms

2) Positive tuberculin skin test

3) History of contact

4) Compatible chest x-ray

5) Bacteriological or histological findings of Tuberculosis (this criterion alone is sufficient for diagnosis) (4).

It is required to change and improve many existing practices such as the ones related to contact investigations and rapid accurate diagnosis to reduce the burden of TB

Copyright (C) 2015, Pediartric Infections Research Center. This is an open-access article distributed under the terms of the Creative Commons Attribution License, which permits unrestricted use, distribution, and reproduction in any medium, provided the original work is properly cited. 
Karimi A et al.

in children. In particular it should be considered that TB control efforts are threatened by the HIV epidemic.

\subsection{Description of Some New Diagnostic Methods}

Recently, in vitro T-cell-based methods, the Tuberculin Skin Test alternative, have been made available to diagnose TB infection; they include interferon $\gamma$ assays (QuantiFERON-TB, Quantiferon- TB Gold, T-SPOT-TB), which use Purified Protein Derivate (PPD), Early Secretory Antigen Target 6 (ESAT6), and Culture Filtrate Protein 10 (CFP10). The latter two antigens are coded by genes of region of difference 1 (RD1) locus of $M$. tuberculosis and are more specific for MT (exception is Mycobacterium marinum, Mycobacterium kansassi and Mycobacterium sulzagi $(1,7,8)$.

\subsection{Methods for Bacilloscopy}

Kinyoun modification, auramine $\mathrm{O}$, and rhodamine stains are used for direct examination of M. tuberculosis $(1,2)$.

\subsection{New Methods for Culture}

However, the gold standard for diagnosis is a positive culture of MT from clinical specimens collected from the suspected cases $(1,9)$. Various culture media used to detect and grow MT include (10):

1) BACTEC radiometric system

2) Mycobacteria growth indicator tube (MGIT)

3) BACTEC MGIT 960 system

4) MB-Redox based on the reduction of a tetrazolium salt indicator, ESP culture system 2 based on the detection of pressure changes and $\mathrm{MB} / \mathrm{BacT}$ system that relies on colorimetric detection of carbon dioxide

5) Phage-based assay based on the ability of MT to support production of mycobacteriphage (like FASTPlaque TB test) $(1,2,8)$.

\subsection{New Methods for Drug Susceptibility Testing}

It is prudent to perform drug susceptibility testing (DST), especially in areas with a high rate of MDR TB. Various methods are devised for DST including the proportion, the resistance ratio, the absolute concentration methods and BACTEC radiometric system divided into genotypic and phenotypic methods. The former include molecular nucleic acid amplification (amplified MT direct test, AMTD and Amplicor MT), real-time Polymerase Chain Reaction (PCR), and strand-displacement amplification methods $(1,2,9,10)$.

The latter include various metabolic tests such as color indicators and oxygen consumption, visualization of micro-colonies, nitrate reduction assay, and use of phages. Examples are Mycobacteria growth indicator tube (MGIT), E-test, MB/bacT system, and ESP culture system $2(1,2,11)$.

\subsection{Molecular Epidemiology Techniques}

Recent advances of molecular epidemiology use techniques which include DNA finger printing, like the restriction fragment length polymorphism, RFLP, spacer oligo- typing and spoligotyping, genomic deletion analysis, and Mycobacterial interspersed repetitive units (MIRU) $(1,2)$.

\subsection{Serological Diagnosis of TB}

The main purpose of these tests is increasing the ability to diagnose latent infection $(1,2)$.

\subsection{Non-Microbiological Diagnostic Techniques}

Detection of adenosine deaminase (ADA) is an easy, rapid, low cost test but can be used as an extra tool for Tuberculosis pleural effusions and meningitis according to its low accuracy $(1,2)$. The major problems encountered in rapid diagnosis of TB and differentiation of infection from the disease includes unavailability of advanced tests and inaccuracy of available tests. In immune compromised patients, especially those with HIV infection, results of diagnostic tests are equivocal, thus making a definite diagnosis becomes even more difficult.

\subsection{Conclusions}

1) In every setting, high index of suspicion is required for rapid diagnosis of $\mathrm{TB}$, and physicians should take the epidemiological, clinical and paraclinical data into account to gauge the probability of active disease to order feasible, accurate and cost effective diagnostic tests.

2) In high endemic areas, TB should be suspected early and steps should be taken for prompt diagnosis and treatment.

3) In every setting, PPD, X-ray, smear, and culture are requested in suspected cases and if available more sensitive tests are required according to the site of infection. In resource-limited countries the priority is to ensure a network of quality-controlled microscopy for AFB in clinical samples, most often sputum.

4) It is recommended to request HIV testing in every child with Tuberculosis.

5) It is better to request drug susceptibility test on all obtained isolates, unless the result of this test in isolates from the index case is available. This policy can help to prevent the emergence of MDR and its transmission.

6) The decision to treat a child should be carefully considered, and once such a decision is made the child should be treated with a full course of therapy.

7) A majority of TB diagnostic studies are focused on test accuracy and not on outcomes such as accuracy of diagnostic algorithms. Revised clinical algorithms for TB diagnosis may demonstrate the impact of these new tools.

\section{Acknowledgements}

The authors wish to thank all the staffs in Pediatric Infections Research Center.

\section{References}

1. Palomino JC. Nonconventional and new methods in the diagnosis of tuberculosis: feasibility and applicability in the field. 
Eur Respir J. 2005;26(2):339-50.

2. Mathur JN. What is New in Diagnosis of Tuberculosis? Part I : Techniques for Diagnosis of Tuberculosis. Indian Council of Medical Research. 2002;32(8).

3. Tuberculosis (TB). WHO; 2014. Available from: http://www.who. int/tb/data.

4. Stop TВPCTBSWHO. Guidance for National Tuberculosis Programmes on the management of tuberculosis in children. Chapter 1: introduction and diagnosis of tuberculosis in children. Int J Tuberc Lung Dis. 2006;10(10):1091-7.

5. MacPherson P, Houben RM, Glynn JR, Corbett EL, Kranzer K. Pretreatment loss to follow-up in tuberculosis patients in low-and lower-middle-income countries and high-burden countries: a systematic review and meta-analysis. Bull World Health Organ. 2014;92(2):126-38.

6. National Collaborating Centre for Chronic Conditions and the Centre for Clinical Practice at NICE,Tuberculosis,Clinical diagnosis and management of tuberculosis, and measures for its prevention and control 2011. 2011. Available from: www.nice.org.uk/nicemedia/ live/13422/53642/53642.pdf.

7. Rhodes S, Holder T, Clifford D, Dexter I, Brewer J, Smith N, et al. Evaluation of gamma interferon and antibody tuberculosis tests in alpacas. Clin Vaccine Immunol. 2012;19(10):1677-83.

8. Simsek H, Alpar S, Ucar N, Aksu F, Ceyhan I, Gozalan A, et al. Comparison of tuberculin skin testing and T-SPOT.TB for diagnosis of latent and active tuberculosis. Jpn J Infect Dis. 2010;63(2):99-102.

9. WHO, Global tuberculosis controlGeneva. World Health Organization. 2011.

10. Dorman SE. New diagnostic tests for tuberculosis: bench, bedside, and beyond. Clin Infect Dis. 2010;50 Suppl 3:S173-7.

11. Moore DA, Evans CA, Gilman RH, Caviedes L, Coronel J, Vivar A, et al. Microscopic-observation drug-susceptibility assay for the diagnosis of TB. N Engl J Med. 2006;355(15):1539-50. 